kann vorübergehend (unstabile Form durch Penicillin) oder definitiv (stabile Form) sein. Im letzteren Fall muß er genetische Ursachen haben; tatsächlich erhält man die Stabilisierung eines Stammes durch laufende Passagen, wobei man Selektion von spontanen Mutanten ausführt. Wie viele solcher Mutationen

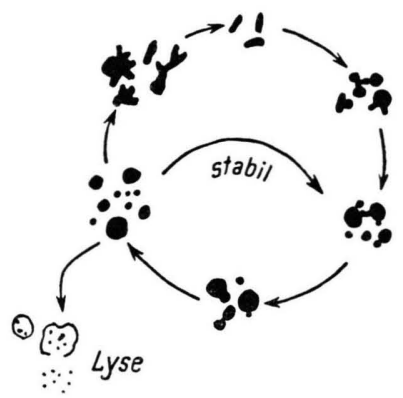

Abb. IV. Schematische Darstellung des nachweisbaren Ablaufs der morphologischen Vorgänge bei stabilen und unstabilen L-Formen.

notwendig sind, ist sehr schwer festzustellen. Sicher müssen es aber mehrere Schritte sein. Inwieweit man in diesem Fall noch eine nahe Verwandtschaft mit dem Ausgangsstamm annehmen darf, können nur eingehende systematische Studien entscheiden.

19 W. Zi m m e r m a n n, Dissertation, Frankfurt a. M. 1954.

$20 \mathrm{~K}$. Li e b e rm e is t e r, Vortrag Tag. Österr. Ges. Mikrobiol. u. Hyg., Innsbruck, Sept. 1954, s. auch Zbl.
Unsere Beobachtungen ergeben, daß der filtrierbare L-Stamm K 52 mit dem PPLO-A-Stamm morphologisch vergleichbar ist; der wesentliche Unterschied der beiden liegt darin, daß der Stamm K 52 ungleichmäßigere Dimensionen zeigt. Man kommt damit der schon öfter geäußerten, aber auch widersprochenen Ansicht einer Verwandtschaft von L-Formen und PPLO-Stämmen wieder näher.

Es ist schwierig, den genauen Teilungsmechanismus der globulären Formen zu beobachten, da die Generationszeit der uns bekannten Stämme sehr groß und die Organismen z. T. sehr klein sind. Eine Sprossung oder eine Zweiteilung scheint am wahrscheinlichsten 19, 20,21. Die Sprossung steht voraussichtlich in engem Zusammenhang mit der Entstehung kleiner filtrierbarer Elemente.

Durch sehr wertvolle techn. Assistenz haben nacheinander Frl. T. B o n n e, Frl. C. G a n g l und Frl. A. R ü te $r$ mitgewirkt.

Die Arbeit wurde ausgeführt mit Unterstützung der Herren Prof. Gras se t und Extermann, der Stif tung Fritz Hoffmann-La Roche zur Förderung von Arbeitsgemeinschaften in der Schweiz und der Deutschen Forschungsgemeinschaft.

Bakteriol., Parasitenkunde, Infektionskrankh., Hyg., I. Abt., Ref. 156, 180 [1955].

21 G. K a nd le r u. O. K a n d l e r, Arch. Mikrobiol. 21, 178 [1954].

\title{
Zur Entwicklungsgeschichte der Plastiden
}

\author{
I. Mitteilung \\ Von Wilhelm MenKe
}

\begin{abstract}
Aus dem Max-Planck-Institut für Biologie, Abt. M e l c h e r s, und dem Max-Planck-Institut für Virusforschung, Tübingen

(Z. Naturforschg. 11 b, 215-216 [1956]; eingegangen am 23. Dezember 1955)
\end{abstract}

Plastiden meristematischer Gewebe besitzen bereits einen Bau, welcher dem der Chloroplasten ähnlich ist.

$F^{i}$ ür die Plastiden meristematischer Zellen höherer Pflanzen konnte bisher kein Autor die typische Lamellar- und Granastruktur der Chloroplasten feststellen ${ }^{1}$. Dagegen besitzen die Chromatophoren der Eizellen von Fucus vesiculosus schon die charakteristische Schichten- und Lamellarstruktur ${ }^{2}$.

1 S. S tr u g g e r, Naturwissenschaften 37, 166 [1950]; Ber. dtsch. bot. Ges. 66, 439 [1953]; Protoplasma 43, 120 [1954]; E. H e it z u. R. M a ly, Z. Naturforschg. 8 b, 243 [1953]; D. D üve l, Protoplasma 44, 239 [1955]; G. Gr a ve, Protoplasma 44, 273 [1955]; J. B ö i n g, Protoplasma 45, 55 [1955]; U. F a s s e-Frantis k e t,
Ich habe chlorophyllfreie Plastiden durch fraktioniertes Zentrifugieren wäßriger Extrakte angereichert, und zwar aus den Vegetationspunkten von Elodea densa, die vor der ersten Blattanlage abgeschnitten wurden, aus Blattanlagen (Länge $\geqq 0,5 \mathrm{~cm}$ )

Protoplasma 45, 194 [1955]; H. L e y o n, Exp. Cell. Res. 5, 520 [1953]; 7, 265 [1954]; 7, 609 [1954]; E. H e itz, Exp. Cell. Res. 7, 606 [1954]; K. M ühle t h a l e r, Protoplasma 45, 264 [1955].

2 H. L e y on u. D. von W e t $t$ s te in, Z. Naturforschg. 9 b, 471 [1954]; D. von Wetts te in, Z. Naturforschg. 9 b, 476 [1954]. 
ruhender und austreibender Zwiebeln von Allium Cepa und aus jungen Blättern von Allium Porrum. Um der Gefahr einer Täuschung durch Artefakte zu begegnen, wurde unfixiertes und fixiertes Gewebe verarbeitet. Im Elektronenmikroskop zeigen diese Plastiden einen Bau, der schon mit der Struktur ausgewachsener Chloroplasten vergleichbar ist, nämlich zahlreiche, in Schichten angeordnete Grana, die anscheinend manchmal schon in Reihen senkrecht übereinander liegen ${ }^{3}$, ferner Granalamellen und Stromalamellen, die beim Zerfall der Plastiden sichtbar werden. Die äußerste Schicht, welche die Plastiden umhüllt, scheint verhältnismäßig derb zu sein und keine Grana zu enthalten. Jedenfalls prägen sich die unter ihr liegenden Grana beim Antrocknen nur undeutlich ab (Abb. $1^{*}$ und 2). Wo diese Schicht jedoch bei der Präparation teilweise oder ganz entfernt wurde, kann man die Grana deutlicher als plättchenförmige Gebilde erkennen (Abb. 3, 4 und 7). Die Lamellen enthalten anscheinend eine einfache Lage korpuskularer Teilchen. Ihre Anordnung in den Präparaten ist manchmal eine gitterartige, die, wenn auch stark gestört, sicher keine hexagonal dichteste Packung ist. Einige Bilder könnte man auch so auffassen, daß noch ein Gitter aus gekreuzten Fibrillen vorhanden ist, auf dem kugelige Teilchen liegen. Es ist aber auch eine andere Deutung möglich. Manche der beim Zerfall freiwerdenden kugelförmigen Teilchen besitzen nämlich einen stäbchenförmigen Anhang (Abb.6). Bei genauerer Betrachtung kann man derartige an Bakteriophagen erinnernde Teilchen auch an vielen Stellen in situ erkennen (Abb. 2, 4 und 5), und man wird untersuchen müssen, ob diese Teilchen die Bauelemente der gitterartigen Strukturen sind.

Von Mitochondrien lassen sich die Plastiden leicht durch ihre Form, Größe und ihren inneren Aufbau unterscheiden. Während die Plastiden als runde Scheiben von 0,6 bis $1,1 \mu$ Durchmesser erscheinen, zeigen die Mitochondrien die Gestalt von Stäbchen mit abgerundeten Enden, deren Länge zwischen 0,5 und $0,9 \mu$ und deren Breite zwischen 0,2 und $0,4 \mu$ schwankt. Damit ist die an sich schon unwahrscheinliche Hypothese, die Plastiden aus Mitochondrien entstehen läßt, noch unwahrscheinlicher geworden.

Fragt man nun, warum andere Forscher die hier aufgedeckten Strukturen nicht gefunden haben, so

3 W. M e n k e, Naturwissenschaften 28, 158 [1940]. kann die Antwort nur lauten, daß diese ungeeignete Methoden angewandt haben. Mit dem Lichtmikroskop kann man Grana, deren Durchmesser kaum $0,1 \mu$ betragen und die ziemlich dicht nebeneinander liegen, überhaupt nicht abbilden. Wenn man jedoch im Elektronenmikroskop Schnitte von Objekten, die mit Osmiumsäure fixiert wurden und aus denen das Einbettungsmittel nicht entfernt wurde, untersucht, so bildet man im wesentlichen die Verteilung der im Präparat entstandenen Osmiumverbindungen ab, weil die meisten Baustoffe des Protoplasmas eine ähnliche Elektronendichte besitzen wie das Einbettungsmittel. Auch die hier angewendete Arbeitsweise hat natürlich ihre begrenzten Möglichkeiten, und man wird weitere Methoden anwenden müssen, um die Ergebnisse auszubauen.

\section{Experimentelles}

Die lebenden Objekte wurden mit 0,5- $m$. Saccharoselösung im Vakuum infiltriert, in einer Porzellanreibschale zerrieben oder zwischen Glasschliffen zerquetscht. Der entstandene Brei wurde mit etwas Saccharoselösung aufgenommen und auf einer Glasfritte (G 2) abgesaugt. Das Filtrat wurde bei 200 bis $300 \mathrm{~g} 10 \mathrm{Min}$. lang zentrifugiert, und aus dem Überstehenden wurden die Plastiden durch 20 Min. langes Zentrifugieren bei 2000 bis $3000 \mathrm{~g}$ sedimentiert und darauf 3-mal auf der Zentrifuge mit destilliertem Wasser gewaschen. Nach hinreichender Verdünnung mit dest. Wasser wurden die Präparate auf Kollodiumfolien aufgetragen und nach Antrocknen mit PlatinRhodium bedampft. Die Aufarbeitung wurde im Kühlraum bei $2^{\circ} \mathrm{C}$ vorgenommen.

Zur Fixierung wurden die Objekte mit 0,5\% Osmiumsäure in dest. Wasser oder in Veronalpuffer $\left(p_{\mathrm{H}}=7,0\right)$ infiltriert und nach 3 bis $16 \mathrm{Stdn}$. in Wasser ausgewaschen. Die Aufarbeitung erfolgte in ähnlicher Weise wie bei unfixiertem Material, nur wurde mit etwas Quarzsand zerrieben und bei Zimmertemperatur gearbeitet.

Zur Prüfung auf Chlorophyll wurden einige Gewebestückchen mit Quarzsand unter Zusatz von Methanol bei roter Dunkelkammerbeleuchtung zerrieben. Das Filtrai wurde darauf mit Benzin ausgezogen, wobei einige Tropfen Wasser zugesetzt wurden. Der Benzinextrakt zeigte nur eine schwache Gelbfärbung und unter der Fluoreszenzlampe eine schwache ziegelrote Fluoreszenz, die auf Protochlorophyll hinweist.

Ich danke Herrn Professor Dr. Melchers für die gastliche Aufnahme in seinem Institut und Herrn Professor Dr.Friedrich-Freks a dafür, daß er das Elektronenmikroskop seines Institutes zur Verfügung stellte. Die Aufnahmen hat Herr B e rge r angefertigt, wofür auch ihm bestens gedankt sei.

* Abb. $1-7$ s. Tafel S. 220 a u. b. 


\title{
Wirkungen von UV- und Röntgenstrahlen auf kernlose und kernhaltige Teile von Acetabularia
}

\author{
Von JoAChim HäMmERLING * \\ Aus dem Max-Planck-Institut für Meeresbiologie Wilhelmshaven \\ (Z. Naturforschg. 11 b, 217-221 [1956]; eingegangen am 13. Dezember 1955)
}

\begin{abstract}
Bereits kurzfristige UV-Bestrahlungen kernloser formbildungsfähiger Vorderstücke von Acetabularia bewirkten durch reine Absorption im Cytoplasma eine starke Senkung des Formbildungsvermögens. Außerdem wurde bei diesen und bei nicht formbildungs-fähigen Teilen die Lebensdauer verkürzt. Bei kernhaltigen Teilen blieb dagegen das Formbildungsvermögen auch bei extremer Strahlenwirkung erhalten. Die wahrscheinlichen Gründe für diesen Unterschied werden erörtert. Irreversible Kernveränderungen wurden bis auf eine fragliche Ausnahme (normale Mutation) bisher nicht beobachtet.

Bei Röntgenbestrahlungen zwischen 6 und $18 \mathrm{kr}$ wurden ausgeprägte plasmatische Dauerschäden weder an kernhaltigen noch kernlosen Zellen gefunden. Eine Analyse von an den Nachkommen auftretenden Abänderungen war nicht möglich. Bei Bestrahlungen von Keimlingen zwischen 120 und $300 \mathrm{kr}$ blieben bei ansteigenden Absterbeziffern immer noch eine Reihe von sich normal entwickelnden Zellen übrig. Erst mit der Kernteilung oder Cystenbildung wurden verschiedengradige Letalitätseffekte manifest, die auf Chromosomenbrüchen beruhen dürften.
\end{abstract}

$\mathrm{D}_{\mathrm{H}}^{\mathrm{s}}$ der Kern der einkernigen Acetabularien im Rhizoid liegt, ist es durch Abschneiden des Rhizoides leicht möglich, kernhaltige und kernlose Teile herzustellen ${ }^{1}$. Es sollte sich deshalb ermitteln lassen, welche Wirkungen von UV- und Röntgenstrahlen auf Absorption nur im Zellplasma oder nur im Kern oder in beiden Zellkomponenten zurückzuführen sind. Dazu kommt der Vorteil, daß kernlose Teile dieser Grünalgen nicht nur lange lebensfähig sind, sondern zum Teil, insbesondere die Vorderstücke, auch ein ausgeprägtes Formbildungsvermögen hinsichtlich Stiel-, Wirtel- und Hutbildung besitzen ${ }^{1,2}$.

Die UV-Bestrahlungen (s. 1-5) wurden mit einer $\mathrm{Hg}$ Hochdrucklampe (Leistung $300 \mathrm{~W}$ ) in einer Entfernung von 12,5 cm ausgeführt. Sie erfolgten in Erdschreiber ${ }^{3}$, und zwar so, daß die Teile gerade noch von Flüssigkeit bedeckt waren. Die Röntgenbestrahlungen (s. 6) wurden unter folgenden Bedingungen ausgeführt: bis $18 \mathrm{kr}$ : $165 \mathrm{kV}, 1 \mathrm{~mm}$ Al-Filter, $6 \mathrm{~m} \mathrm{~A}$, Dosisleistung rd. 130 r/min; ab $120 \mathrm{kr}$ : $170 \mathrm{kV} 1 \mathrm{~mm}$ Al-Filter, $8 \mathrm{~m} \mathrm{~A}$, Dosisleistung (durch geringeren Abstand) rd. $1200 \mathrm{r} / \mathrm{min}$. Die Bestrahlung erfolgte auf feuchtem Fließpapier. - Die Kontrollen wurden in allen Einzelheiten wie die bestrahlten Teile behandelt (was unerläßlich ist, da bereits die für die Bestrahlungen vorgenommenen Manipulationen als solche zum Teil Schädigungen hervorriefen).

* Mit Unterstützung durch die Deutsche For $\mathrm{schungsgemeinschaft}$.

1 J. H ̈̈m m e r ling, Roux' Arch. Entwicklungsmechan. Organismen 131, 1 [1934]; Biol. Zbl. 54, 650 [1934].
1. Bei UV-Bestrahlung von $1 \mathrm{~cm}$ langen Vorderstücken der Art A.mediterranea, die von kurz vor der Hutbildung stehenden Pflanzen stammten, nahm das Formbildungsvermögen bereits nach einer Be-

\begin{tabular}{|l|c|rr|r|l|}
\hline & & \multicolumn{2}{|c|}{$\begin{array}{c}\text { Stielzuwachs } \\
\text { pro Teil }\end{array}$} & \multicolumn{2}{|c|}{ Bildung von } \\
& $n$ & Wirteln & Hüten abs. \\
& & {$[\mathrm{mm}]$} & {$[\%]$} & pro Teil & Zahlen \\
\hline Kontr. & 20 & 2,6 & 100 & 1,5 & 6 typ. \\
$1^{\prime}$ bestr. & 24 & 1,7 & 65 & $0,5^{1}$ & 1 Kümmerh. \\
$2,5^{\prime}$, & 22 & 0,7 & 27 & 0 & 0 \\
$5^{\prime} \%$ & 16 & 0,4 & 15 & 0 & 0 \\
\hline
\end{tabular}

$1 \mathrm{Da}$ vielleicht einige alte Wirtel als neugebildet angesehen wurden, sank die Wirtelbildungs-Fähigkeit bei den 1 Min. lang bestrahlten Teilen möglicherweise nur um 50 Prozent.

Tab. 1. Leistungen uv-bestrahlter kernloser $1 \mathrm{~cm}$ langer Vorderstücke von Acetabularia mediterranea.

strahlungszeit von 1 Min. erheblich ab und sank bei Erhöhung der Strahlendosis weiter (Tab. 1). Hinsichtlich des Stielzuwachses scheint ein exponentiell verlaufender Abfall vorzuliegen. Am stärksten betroffen wurden die Hutbildungsprozesse, also diejenigen Vorgänge, die nach allen Erfahrungen eine beson-

2 J. Hämmerling, Int. Rev. Cytology 2, 475 [1953].

3 J. Hämmerling, Arch. Protistenkunde 97, 7 [1944]. 